far as my own two cases are concerned I do not think that the reduction in either of them, great as it was, was more than could reasonably be accounted for in this way. In most, however, there was another, and perhaps a more important, factor in bringing about this result. It has been shown by Griffiths, ${ }^{6}$ and it has been confirmed by my own investigations and those of others, that, while the primary hange in enlargement of the prostate is a form of glandular overgrowth, fibroid degeneration, secondary to this, always follows at a very early period in some cases, leading to an exceedingly dense mass of scirrhous hardness, and much later in others ; and I think it is allowable to infer that this fibroid transformation, if it has not already commenced, might be started from foci of cicatricial contraction following suppuration. Certainly the method employed in nearly all these cases was such as would cause suppuration and tolerably extensive cicatrisation afterwards. The maintenance of a cannula, for example, for many weeks together in the middle of an enlarged gland, or puncture with a galvano"cautery or the removal of portions of the mass through a supra-pubic opening in a bladder affected with intense cystitis, would undoubtedly lead to this. It is to this, I believe, that the benefit that has undoubtedly been experienced in many of these cases is due; and this, too, affords a reasonable explanation of the fact that the result in (unfortunately) the greater number has been negative-the fibroid transformation was already so far advanced in them that further contraction could not take place.

There is no evidence, so far as I have been able to ascertain, that atrophy has ever been induced in this way. True atrophy does occur after castration. The gland shrinks to a small fibrous nodule. But it is not said in any case that I have discovered that the prostate contracted to less than its normal size. All that is said is that the gland diminished to sach an extent that nothing that was abnormal could be feit per rectum, or that in some the obstruction to micturition, in others the obstacle that prevented the passage of a catheter, almost disappeared ; and this is not sufficient.

There are certain corollaries of some importance that follow from this.

1. Prostatectomy should not be performed shortly after an attack of acute retention. It is not possible, without waiting a little, to say how much of the enlargement is due to congestion, and the hæmorrhage may be severe.

2. In cases of septic cystitis it may be advisable to perform the operation in two stages. First the bladder should be arained by supra-pubic cystotomy, then after a few days, when the congestion has subsided and the urine has lost its foul and septic character, the obstruction at the neck should be dealt with. It is conceivable from the amount of shrinkage that has taken place that in some cases the second part of the operation may not be required, and there would be at least this advantage that the wound left after the growth had been excised-always a very ragged onewould not be bathed in putrid urine.

3. If prostatectomy, with the view of establishing a lowlevel urethra, is thought advisable after the congestion has subsided, the operation, as I mentioned in my lectures, should be thorough. Every atom of the obstruction then must be due to the growth. This is especially necessary where, as in Mr. Gilbert Barling's case, the prostate is very hard. If the overgrowth has alrcady passed into the stage of fibroid induration, it cannot contract any more. It does not follow, however, that if the prostate is soft and adenomatous the portion removed should be a small one, in the hope that condensation and contraction would do the rest.

4. The merits of prostatotomy are not due to the division of the gro wth, but to the changes induced by subsequent drainage.

Wimpole-street, $\mathrm{W}$.

\section{A CASE OF CIRRHOSIS OF THE LIVER PRESENTING SOME UNUSUAL SYMPTOMS.}

BY H. W. WEBBER, M.D. \& B.S. LOND.

A PUBLIC-HOUSE-KEEPER aged fifty-one was seen first on Fov. 11th, 1893, complaining of abdominal pain and dissomfort, with flatulence, nausea and a little sickness, and pain in the lower parts of the back. There had been con-

6 Journal of Anatomy and Physiology, 1888, 1889, 1890. stipation for two days; no headache was complained of. The tongue was coated, the pulse 90 , and the temperature $100.8^{\circ} \mathrm{F}$. The patient had been a beavy drinker in former years, and had continued this habit, but with more moderation, latterly. He was ordered an effervescing mixture and low diet. On the 12th the bowels acted slightly and the temperature was $101^{\circ}$. On examination of the abdomen the liver dulness was found to commence above at the lower border of the fifth rib, while below the edge of the liver could be indistinctly felt at a distance of rather more than an inch from the costal margin; no tenderness of the liver was felt on deep pressure. The abdominal walls were fatty, and scattered over the skin of the abdomen and lower part of the chest a few dilated bloodvessels were present. The edge of the spleen was not palpalole or the splenic dulness increased; no peritoneal exudation was present. On examination numerous dilated vessels and petechial papules were found in the skin over the face, arms, and back. On the 15th the temperature was $100^{\circ}$, and pain in the back was still complained of, but the nausea had ceased. The bowels now acted once or twice a day ; the urine was high-coloured and contained lithates, but no albumen. On the 19th the patient suffered slightly from epistaxis. The respiration was somewhat laboured and wheezy, though no adrentitious sounds were, heard on auscultation, nor was there any expectoration. He was now ordered a mixture containing potassium citrate, salicylate of soda, and ammonium acetate every four hours, and was put on slop diet. On the 23rd the temperature, which had been $100^{\circ}$ for two days, rose to $103^{\circ}$, the patient's face was flushed and the eyes injected, the tongue was coated, and the pulse 120 . The oozing from the nares still continued. The motions were of the colour and consistence of putty; the urine was high coloured, depositing lithates. The edge of the liver was palpable, more distinctly than at first, two inches below the costal margin, but only slightly tender on deep pressure. He was ordered quinine sulphate (one grain) in pill form every four hours, with the previous mixture. On the 24 th the temperature fell to $100^{\circ}$, only to rise the day after to $102^{\circ}$. On the 26th it had fallen to $99^{\circ}$, but on the 27 th it was $103^{\circ}$. On the latter day the patient had profuse epistaxis, and complained of headache. The bowels continued to act rather irregularly, but generally at least one motion was passed every day; it was of the same character as noted previously. No reaction for bile could be obtained from the urine, though the tests for this were applied on several occasions. The patient was now treated with a mixture containing chlorate of potash and Warburg's tincture given every four hours, with a dose of castor-oil when required to assist the action of the bowels. During the early part of December the condition very gradually improved, and the temperature kept generally at a much lower level. There was epistaxis at times, though not profuse. In the third week of December he was able to be about a little. This improvement, however, only lasted for a few days, and on Dec. 25 th he had to take to his bed again. His condition at this time was as follows : the face was fiushed and the conjunctival vessels somewhat dilated there was no jaundice. The skin over the body generally was of a pale lemon or primrose tint, with numerous scattered papular petechial spots. Blood oozed occasion. ally from the nose. The tongue was coated on the dorsum and red at the edges. The whole of the fauces and pharynx were intensely red and covered with yellowish stringy mucus. The patient was tronbled at times with fits of coughing, when be would expectorate a little frothy blood; he was constantly hoarse. No abnormal physical signs were discovered in the chest. The edge of the liver could be felt at a distance of three inches from the costal margin, rounded, the viscus now being distinctly tender. There was no enlargement of the spleen nor any signs of fluid in the peritoneal cavity. The cardiac sounds presented nothing abnormal ; the pulse was generally 120 , full, soft, and of medium tension, the temperature varying from $99^{\circ}$ to $1002^{\circ}$ in the morning and from $101^{\circ}$ to $102.4^{\circ}$ in the evening. The throat was painted with borax and glycerine and an opiate linctus given to relieve the cough. On Dec. 30th a small percentage of albumen was found in the urine. On the 31st the patient had an attack of bleeding from the left nostril, which was so profuse that it was plugged with strips of lint soaked in hazeline. On the evening of Jan. 1st, 1894, there was a discharge of blood-stained fluid from the left ear. On the 2nd profuse hæmorrhage, requiring plugging, took place from the right nostril, and on the 3rd there was a blood-stained discharge from the right ear. No albumen was found in the 
urine on the 4th and none was found afterwards. Discharge of sanguineous fluid now continued fairly profusely from both ears, so that unless intercepted it would run on to the pillow. This continued constantly, though to no great amount, until Jan. 15th, when there was a profuse hæmorrhage from the left ear. This did not recur again, and the discharge gradually became purulent in character. The ears were syringed gently twice a day with a weak solution of Sanitas Guid, and the discharge received on sal-alembroth wool. The inflammatory condition of the fauces and pharynx gradually subsided, but about this time the gums became swollen, spongy, and purpuric, blood constantly oozing in small quantities. On Jan. 29th the patient was seen in consultation with Dr. Goodhart, who diagnosed the case as one of cirrhosis of the liver, the symptoms, however, being unusual. Treatment with Warburg's tincture was resumed, and an aperient mixture of magnesium sulphate ordered every morning, if necessary, to maintain a free action of the bowels. The patient now began gradually to improve, the temperature reaching normal in the morning, with a slight rise in the evening, by the second week in February. Slight oozing from the nostrils took place at times, the gums continued rather hæmorrhagic, and purulent discharge persisted from both ears; but the patient slowly gained strength. No œdema of the legs was present at any period of the case, nor was there any hæmatemesis or melæna. On March 24th the condition was as follows: there was a purulent discharge from both ears (on two or three occasions there had been slight tenderness over one or other mastoid process, lasting only for a short time, however), sometimes there was slight oozing from the nostrils. 'The gums were a little spongy and bled occasionally, but were much firmer, an astringent mouth-wash having been used. The faucial macous membrane was still a little reddened. The skin presented numerous dilated vascular spots. The liver reached three inches below the costal margin and was only very slightly tender on deep pressure. The pulse was usually about 85 and the temperature was normal both morning and evening. The case was attended in conjunction with my partner, Mr. A. E. Garrett, to whom I am indebted for much information.

The case is of interest from the presence and persistence of three rather unusual symptoms: (1) hæmorrhage from the gums; (2) hæmoptysis; and (3) pyrexia. I am unable to find mention of bleeding from the gums in any of the textbooks which I have examined, and, as will be seen from the result of an analysis of recorded cases below, it is extremely rare. With regard to the hamoptysis, it may be questioned whether the source of the blood was not in the throat; but the fact that the patient was hoarse and at times wheezy, and that he had been often observed to have a distinct fit of coughing resulting in, and relieved by, expectoration of a fairly considerable amount of bright red frothy blood, renders it, I think, clear that a large part of the blood proceeded from the mucous membrane of the lungs. The presence of pyrexia in cirrhosis of the liver is not a very uncommon complication, but it is certainly unusual to find it of such long duration as in the present case. Epistaxis is also not an infrequent symptom, but I think that the persistent oozing with occa. sional sharp bursts of hæmorrhage met with in this case is not often seen.

In a series of fifty cases of cirrhosis of the liver in Guy's Hospital during the years 1891 and 1892, in one only was hrmorrhage from the gums noted; in this case the amount was small and it lasted about a fortnight. In three cases the expectoration was tinged with blood during some part of the time the patients were under observation, and in one case frothy blood was frequently expectorated in small quantities, but it seemed uncertain whether its source was not in the throat. Cases in which concurrent disease in the lungs was present have not been included. Epistaxis was reported in two cases only of the series, and in both these the amount of hæmorrhage was moderate. Pyrexia was present in nine cases $;$ in three of these the temperature rose to $103^{\circ}$, in one of which the pyrexia was of an intermittent type and sasted for three weeks, in the other two the duration was for one week, it falling gradually. In five cases elevation of temperature to $100^{\circ}$ took place on more than one occasion; in one case the temperature rose once to $101^{\circ}$, and more than once to $100^{\circ}$.

There remains one remarkable symptom to be noticed, the bxmorrbage from the ears, which would appear to have arisen by extension of inflammation through the Eustachian tabe from the throat to the middle ear, with perforation of the membrana tympani.

\section{THE TREATMENT OF SEA-SICKNESS.}

By M. CHARTERIS, M.D., L.R.C.S. EdiN. \&c., PROFESSOR OF MATERIA MEDICA AND THERAPEUTICS, UNIVERSITY OF GLASGOW.

In fulfilment of a promise made in a communication to THE LANCET of Feb. 15th, 1893, and in response to many inquiries, I now desire to place before the profession some facts which justify the statement which I made, "that chlorobrom, judiciously taken, will prevent an attack of seasickness or cut it short, if it has already begun, even in those most predisposed to this terror of ocean travellers." These facts are mainly based upon reports of ship surgeons in medical charge of steamers sailing between Great Britain and the United States, Canada, India, Australia, and New Zealand; but before proceeding to do this I consider it advisable to give a few introductory remarks on the nature of sea-sickness and on the remedies which at various times have been adrocated as curative agents in this malady.

Nature of sea-sickness. - Personal experience or observation of those who are afflicted with sea-sickness shows convincingly the "quite, quite down feeling" of Shakespeare, which culminates sooner or later (after a short or more prolonged period) in vomiting, as a rule with little effort. The stomach is the master of the situation, and its contents are ejected. After this the erect position cannot be maintained, and the sick person is forced to lie down. Repeated romiting is succeeded by distressing retching and mental and physical prostration, and the patient now lies half asleep, half awake, and is visited by dreams which are not pleasant. Vomiting, we know, can be excited by direct irritation of the stomach or of the vomiting centre in the medulla oblongata. Many laboured theories have been advanced as to which of these is the primary cause in the romiting of sea-sickness. It would be an unprofitable task to discuss the arguments which have been brought forward. I believe that vomiting is due to gastric disturbance, and that the retching which follows vomiting depends on irritation of the vomiting centre. This conclusion will be more fully supported when we come to consider the treatment of sea-sickness.

Remedies tried in sea-sickness. - If we turn to non-medical writers we find that Bacon extolled a liver pad and a light binder over the abdomen to prevent the churning intestinal movements. Shakespeare was not a medical man, but his works often show that the ars medendi was not outside his ken. In "Cymbeline" he relates how the fair Imogen, before undertaking the journey to Italy to rejoin her lover, received a certain cordial with this advice: "If you are ill at sea, a dram of this will drive away all distemper." The heroine had not the opportunity of testing its efficacy in mal de mer, but when wearied by troubles on land she had recourse to the soothing draught, and sleep long and sound followed. Doubtless this draught contained some narcotic, but what this was the great dramatist has not revealed. Long ago green midshipmen in the English navy had a rope's-end applied to them to stir them up to their duties, and sea-sick men on board whaling vessels had buckets of salt water dashed over them. Boys on board training ships when sick were supported in each case by two persons, and had to toe the deck and to swallow occasionally spoonfuls of hot soup until they recovered. In recent years Dr. Chapman has recommended rest and the application of an ice-bag to the spine. He advised this because he considered that sea-sickness depended upon cerebro-spinal, or rather spinal, congestion; cocaine, nitro-glycerine, nitrite of amyl, atropine, and antipyrin have also had their advocates. Experience has, however, shown that the effect of any of these agents secures no permanent recovery. The only remedy which possessed a curative action seemed to be bromide of potassium, but its advantages were neutralised by its bitter taste and its liability to be rejected by the stomach when given in sea-sickness. Full details were published in THE LANCET of March 5th, 1892, of the steps which led me to combine it with chloralamide, and it is unnecessary to state them again. It is sufficient to say that the solution chlorobrom is stable, palatable, and safe. From 300 cases reported by thirty ship surgeons I shall give some extracts as to its utility.

Testimony of ship surgeons.-Dr. Belcher, of the Cunard 